\title{
Tumefactive Virchow Robin Spaces and its Mimics in Paediatric Patients: A Pictorial Review
}

\author{
Bhavini Gupta ${ }^{1 *}$, Sumit Pruthi ${ }^{2}$, Omprakash Tavri ${ }^{1}$ and Sarfaraz Shaikh ${ }^{1}$ \\ ${ }^{1}$ Department of Radiology, Dr. D.Y. Patil University School of Medicine, India \\ ${ }^{2}$ Vanderbilt Children's Hospital, Nashville, Tennessee, USA \\ *Corresponding Author: Bhavini Gupta, Department of Radiology, Dr. D.Y. Patil \\ University School of Medicine, India.
}

Received: April 14, 2021

Published: May 20, 2021

(C) All rights are reserved by Bhavini Gupta., et al.

\begin{abstract}
Virchow Robin spaces represent pial-lined perivascular spaces and correspond to extensions of subarachnoid space, found in certain characteristic locations around the brain. These spaces are predominantly filled with interstitial fluid and follow CSF intensity on imaging. Enlarged Virchow-Robin spaces, also known as enlarged perivascular spaces (EPVS) are considered when their size increases beyond $2 \mathrm{~mm}$.

EPVS can have varied appearance and may appear in varying locations, making diagnosis difficult. They may be found in normal young individuals, in the mesencephalon, hippocampus and basal ganglia regions, but are usually associated with lower cognition in the healthy older population. Among the younger patients, EPVS are associated with inflammatory and metabolic disorders. While EPVS is common, it is generally overlooked due to their uncertain pathophysiology.

The current review aims to evaluate and characterize different pathologies associated with enlarged and prominent Virchow-Robin spaces and demonstrate the uncommon presentations of common entities that mimic prominent VR spaces. Due to the lack of clinical data, a large proportion of the cases presented in this study are misdiagnosed as some of their commoner counterparts, limning the need to outline characteristic features of these diseases. This will help to tailor treatment appropriately and help the patients who suffer from these disorders.

Presenting cases of enlarged bilateral tumefactive VR spaces were studied retrospectively, including their biochemical, genetic, and clinical features, to finally arrive at a diagnosis.

Keywords: Tumefactive VR Spaces; Virchow-Robin Spaces; Neuroradiology; Periventricular Cysts
\end{abstract}

\section{Abbreviations}

VR: Virchow-Robin Spaces; PVS: Perivascular Spaces; EPVS: Enlarged Perivascular Spaces; CSF: Cerebrospinal Fluid

\section{Introduction}

The perivascular spaces, as their name suggests, also known as the Virchow-Robinson spaces, are pial lined interstitial fluid filled structures that accompany penetrating cerebral vessels for variable distances, as they descend into cerebral substance [1-3]. Electron microscopy and tracer imaging shows no communication with the subarachnoid space [3,6,7]. VR spaces of intracortical arteries is in direct continuity with VR spaces of the subarachnoid space. Lack of leptomeningeal coating around veins in the cerebral cortex, suggests that VR spaces around the vein are in continuity with subpial space [7]. Tracer studies and pathological analysis of the brain also show that VR spaces carry interstitial solutes from the brain and form the lymphatic drainage pathways of the brain [8]. Though believed to occur more commonly in older patients, recent evidence suggest that perivascular spaces can be identified on MR imaging obtained in patients of all ages [4]. 


\section{Materials and Methods}

MRI Brain scans carried out in the hospital were investigated and analysed for the presence of tumefactive Virchow-Robin spaces. These patients were then followed-up retrospectively, for final diagnosis of their neurological diseases. The various differentials found are outlined below, in this review.

Axial T2W images were used for the evaluation of prominent VR spaces. Lack of FLAIR hyperintense rim was taken as evidence of VR spaces to differentiate from gliotic and ischemic changes.

\section{Imaging of VR spaces}

VR spaces are well-defined CSF intensity structures, with smooth margins, occurring randomly in varying locations within the brain, commonly in the thalamus, basal ganglia, mesencephalon, and the hippocampus [44]. Since, the VR spaces form communicating compartments, no restriction on diffusion weighted images is noted.

No enhancement on post contrast study is noted, helping radiologists to differentiate it from other pathologies. As opposed to other pathologies, tumefactive VR spaces, if moderately enlarged, shows no surrounding changes in the brain parenchyma, which shows normal signal intensity $[15,16]$.

Being similar to CSF, the VR spaces appear similar to CSF intensity on all pulse sequences. However, the signal intensities of the VR spaces are generally lower than that of the CSF containing structures within and around the brain, consistent with the fact that VR spaces represent entrapped interstitial fluid [1]. PVSs are characteristically divided into three subtypes based on location. Type I PVSs appear along lenticulostriate arteries entering the basal ganglia through the anterior-perforated substance. Type II PVSs are found along the path of perforating medullary arteries as they enter cortical grey matter over the high convexities, extending into the white matter [21]. Type III PVSs appear in the midbrain, at the pontomesencephalic junction, surrounding the branches of collicular and accessory collicular arteries [44]. However, they can be found throughout the infratentorial and supratentorial brain, along the vessels.
Different theories have been postulated to explain the enlargement of VR spaces: segmental necrotizing angiitis of the arteries or increased permeability of the arterial wall [11-13], disturbance of drainage of interstitial fluid due to CSF circulation in the cisterns $[9,10]$, spiral elongation of the blood vessels and cerebral atrophy resulting in a prominence of tunnels filled with extracellular fluid $[12,14]$, gradual leaking of interstitial fluid from intracellular compartment to the pial space [14] or fibrosis or obstruction of the VR spaces along the length of arteries and consequent impedance of fluid flow [3]. They are considered enlarged when their size exceeds $2 \mathrm{~mm}$ and has irregular shape [19,20,44].

Occasionally, the VR spaces may become quite enlarged, causing mass effect, assuming bizarre configurations and producing neurological symptoms [5]. These can be mistaken for ominous pathologies, such as cystic neoplasms.

The majority of studies presenting evidence of tumefactive VR spaces, report single, scattered cases or delineate broad clinical and imaging spectrum. This purpose of this review is to present specific imaging diagnosis, verified by genetic and chromosomal testing, that help to characterize tumefactive VR spaces. As opposed to older patients, most of the cases presented in our study are young adults and paediatric patients with varying neurological deficits. A combination of clinical examination, genetic and laboratory testing with body imaging were used to make the final diagnosis.

Pathological entities mimicking and involving perivascular spaces

\section{Hypomelanosis of Ito (HI)}

Hypomelanosis of Ito is the third most common neurocutaneous syndrome [17], the other two being Neurofibromatosis and Tuberous sclerosis. It is thought to be unknown aetiology, however some attribute it to the chromosomal instability - mostly mosaicism [18] and some attribute it to the autosomal dominant inheritance.

Characteristics of $\mathrm{HI}$ are hypopigmented areas with irregular borders, streaks, whorls, or patches and it is commonly associated with neurological abnormalities. 
Neurological manifestations include microcephaly or macrocephaly, cognitive and motor retardation, seizures, ataxia, hyperkinesia and hypotonia, and motor disturbances [22].

On imaging, certain characteristic radiologic features are seen, including hemi-megalencephaly, cortical malformations, bilateral dilated Virchow-Robin spaces, brain atrophy, small discrete bilateral periventricular cysts, abnormal white matter signal, grey matter heterotopias and neuronal migration defects. It has an association with medulloblastoma [23]. The dilated perivascular spaces follow CSF intensity on all MRI sequences with absence of FLAIR hyperintense rim (Figure 1).

Following a brain MRI, clinical evaluation will help us to confirm the diagnosis. Clinically, patients present with hypopigmented linear patches over the appendiceal limbs, known as the Hypo-lines of Ito. Facial features show abnormal dentition, dysmorphic facial features and prominent jaw (Figure 2).

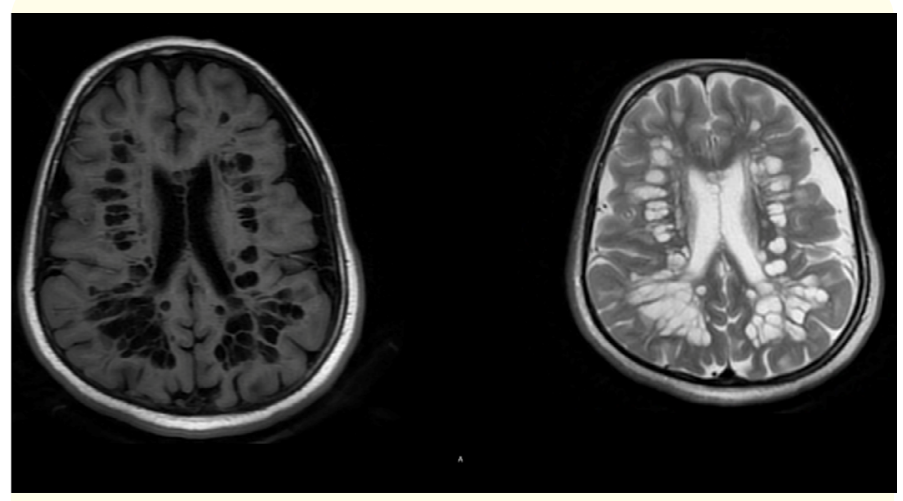

Figure 1: FLAIR and T2W axial images showing multiple, variable sized cystic lesion in the periventricular region, with loss of brain parenchyma. The cysts follow CSF intensity, appearing hyperintense on T2W and hypointense on FLAIR images.

\section{Cystic encephalomalacia}

Multicystic encephalomalacia is a rare cerebral disorder seen in premature neonates secondary to intrauterine placental insufficiency, birth asphyxia, perinatal cerebrovascular accident or neurological trauma $[24,25]$. In this condition, significant portions of

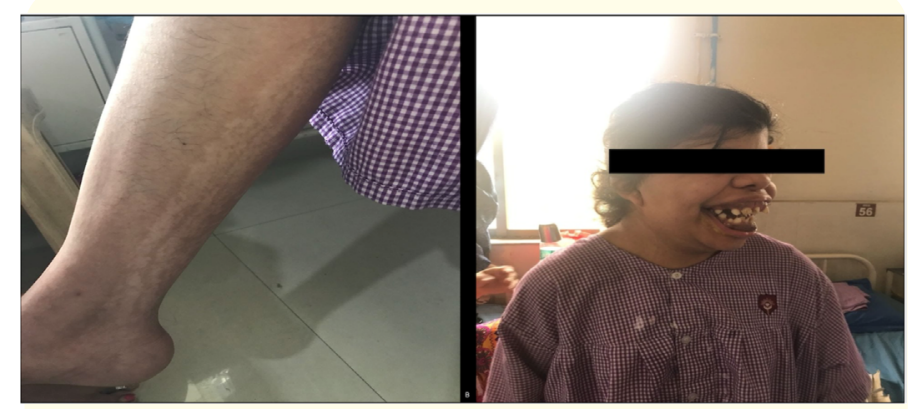

Figure 2: Clinical pictures of the same patient showing multiple hypopigmented linear patches over the shins. Facial features show abnormal dentition, dysmorphic facial features and prognathism.

the brain are replaced by numerous loculated cysts in the white matter and cortex [24].

When caused by thromboembolic infarction, these lesions usually occur along the vascular territories of anterior cerebral artery and middle cerebral artery with sparing of the temporal lobes, posterior fossa, thalami, and deep grey matter [26]. When they occur secondary to birth asphyxia, involvement of cortex and peripheral white matter, primarily in the watershed areas is seen [25]. Neonates with this disorder, tend to have a poor prognosis with neuronal and motor deterioration.

Cystic encephalomalacia is characterized by multiple, variable sized cysts, predominantly involving the inner layers of the cortex, with relative sparing of the cerebellum and the spinal cord, and certain supratentorial structures like the basal ganglia, temporal lobes and the orbital surfaces of the frontal lobes [38]. These cysts appear hypointense on T1W and FLAIR sequences and hyperintense on T2W images. Loss of cerebral parenchymal with ex-vacuo dilatation of the bilateral lateral ventricles (Figure 3 and 4).

\section{Cystic periventricular leukomalacia}

PVL is defined as ischemic infarction, and subsequent white matter injury, due to hypoperfusion of the end arteries that supply the white matter located around lateral ventricles of the premature infant brain [28]. The affected white matter undergoes congestion and necrosis secondary to ischemic insults, followed by cavitation 


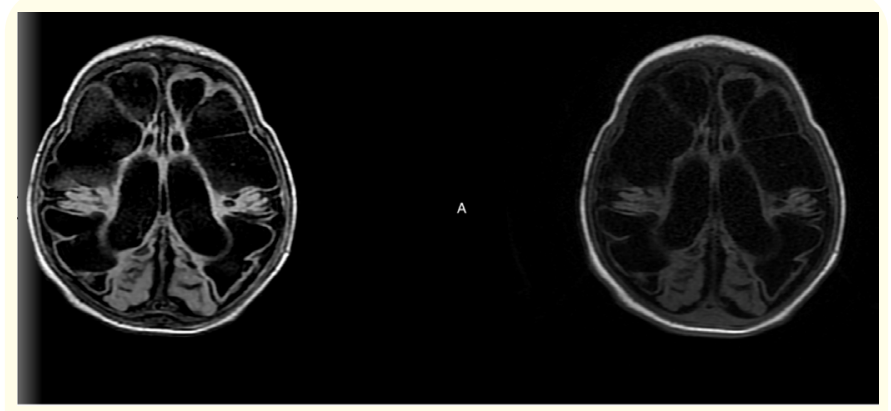

Figure 3: MRI brain of premature infant with birth asphyxia. FLAIR and T1W images show hypointense large cortical cysts with glial septations in bilateral cerebral hemispheres. There is ex-vacuo dilatation of the lateral ventricles. Loss of cerebral volume is noted.

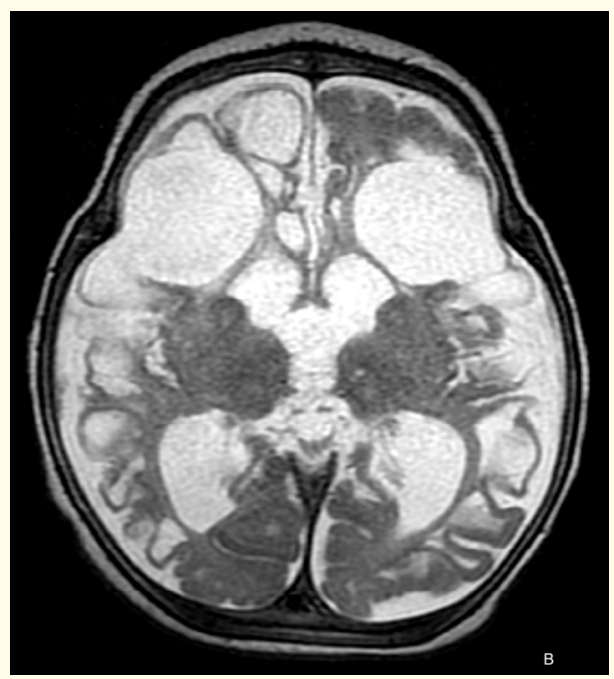

Figure 4: T2W images show hyperintense cysts. Sparing of the periventricular white matter and the deep grey nuclei can be appreciated.

within the necrotic areas. It is a major neuropathological form of brain injury, causing significant neurological morbidity in survivors of premature birth [27]. Common clinical presentations include cerebral palsy, spastic quadriplegia, cortical blindness, deafness and intellectual disturbances [27]. Cranial ultrasonography is at the forefront for diagnosis and follow up [29].

Based on MRI, it has 6 grades:
- Grade 1: Normal MR findings

- Grade 2: Increased signal on long TR sequences.

- Grade 3: Few punctate hemorrhages in white matter.

- Grade 4: Multiple (more than 6) areas of punctate hemorrhages in white matter or larger hemorrhages, less than 2 in number.

- Grade 5: Extensive changes of increased signal on long TR sequences with areas of punctate or focal hemorrhages.

- Grade 6: Diffuse signal intensity changes with hemorrhagic lesions or cystic lesions involving both periventricular and subcortical white matter [30].

There is periventricular white matter necrosis, followed by cavitation and cyst formation, and loss of brain parenchyma.

On imaging, presence of irregular outline of ventricular walls and thinning and bowing of corpus callosum, with sparing of the cortical mantle, characterize the severity of the hypoxic brain injury. There is significant loss of white matter with compensatory enlargement of the ventricles. The involvement tends to be symmetrical [21]. Large Virchow Robin spaces found can confound diagnoses, but are usually not associated with neurological impairment, as seen with PVL (Figure 5a and 5b).

\section{Gliotic areas}

Gliosis is focal proliferation of glial cells in the central nervous system in response to an insult [31]. It is not synonymous with encephalomalacia, an end result of liquefactive necrosis of brain parenchyma [31]. Astrocytes and microglia are glial cells responsible for tissue response to injury.

Gliosis appears bright on T2W images and hypointense on FLAIR and T1W sequences. A FLAIR hyperintense rim around the gliotic areas differentiates it from perivascular spaces, and is characteristic for gliosis (Figure 6).

\section{VRS expansion}

Expanded VR spaces are better diagnosed using Brain MRI, and are noteworthy differentials of lacunar infarcts [2,15,32-35]. They are commonly found in the lower third of basal ganglia, corona radiata, centrum semiovale, hippocampus, insula and midbrain 

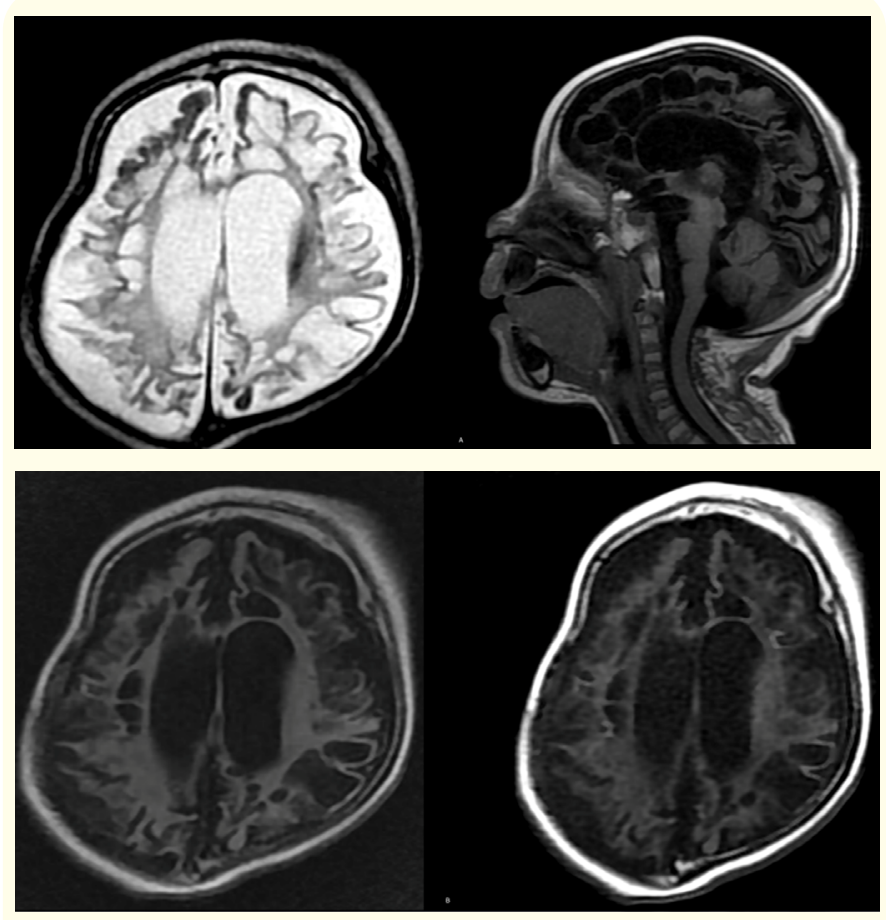

Figure 5a and 5b: Preterm neonate with difficult birth history with late stage periventricular leukomalacia. T2W axial and $\mathrm{T} 1 \mathrm{~W}$ sagittal images; axial T1W and FLAIR images showing T1W and FLAIR hypointense and T2W hyperintense large cystic VR spaces involving the periventricular white matter with irregular outline of lateral ventricular wall. Ventricular enlargement and loss of cerebral volume is prominent. Bilateral subdural hygromas. T2W images show minimal signal changes in the left white matter tracts. Ventricular enlargement with irregular ventricular outline is noted. There is prominent cerebral volume loss. Relative sparing of the grey matter is seen.

[2,15,32-35], with majority in the ponto-mesencephalic junction. Most likely, these expanding VR spaces are caused due to disturbance in the drainage route of the interstitial fluid due to CSF circulation in the cistern $[9,10]$.

To define VR spaces on MR imaging, their distribution should conform to the path of penetrating arteries [2,15,32-35]. In the upper midbrain, enlarged VR spaces are found along the distribution of posterior thalamo-perforating artery or the paramedian

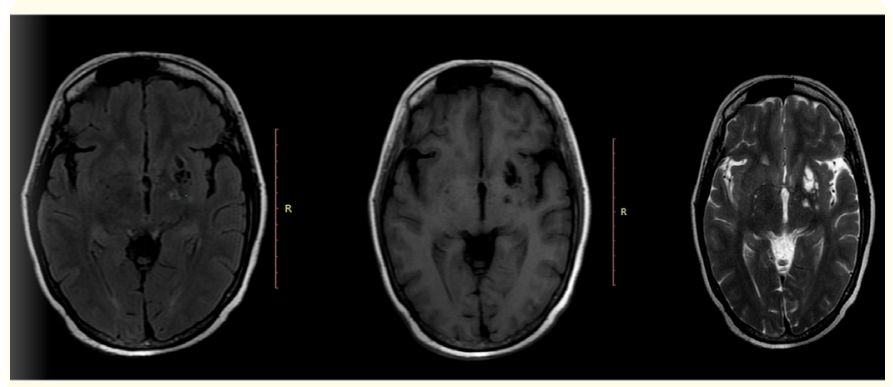

Figure 6: An adult lady came with episode of weakness. Axial FLAIR, T1W and T2W images respectively, show well-defined gliotic areas in the left putamen, anterior limb of left internal capsule and left external capsule with FLAIR hyperintense rim. FLAIR images also show periventricular white matter hyperintensities suggestive of ischemic changes, favouring diagnosis of old ischemic insult.

mesencephlo-thalamic artery, and short and long circumferential arteries originating from upper basilar or proximal posterior cerebral artery [36,37]. No mass effect or neurological defect is usually appreciated in these patients. Absence of enhancement helps to differentiate it from infective etiologies (Figure 7a and 7b).

\section{Neurocysticercosis and tuberculomas}

\section{Neurocysticercosis}

A common infection, caused by the parasite Taenia solium. Tiny fluid filled cysts, generally with an internal scolex is noted, typically in the grey-white matter junction, basal ganglia, cerebellum and brainstem, and subarachnoid spaces, ventricles or spinal cord [21].

Imaging appearances vary depending on the stage of the larval development (evolution), and four stages have been described viz. vesicular, colloidal vesicular, granular nodular and nodular calcified.

In the vesicular stages, fluid-filled cysts, following CSF intensity with an eccentric scolex within is seen. The lesions do not enhance. In the colloidal vesicular stages, peripheral enhancement of lesions and the scolex within is noted. Significant surrounding oedema can be seen (Figure 8). 

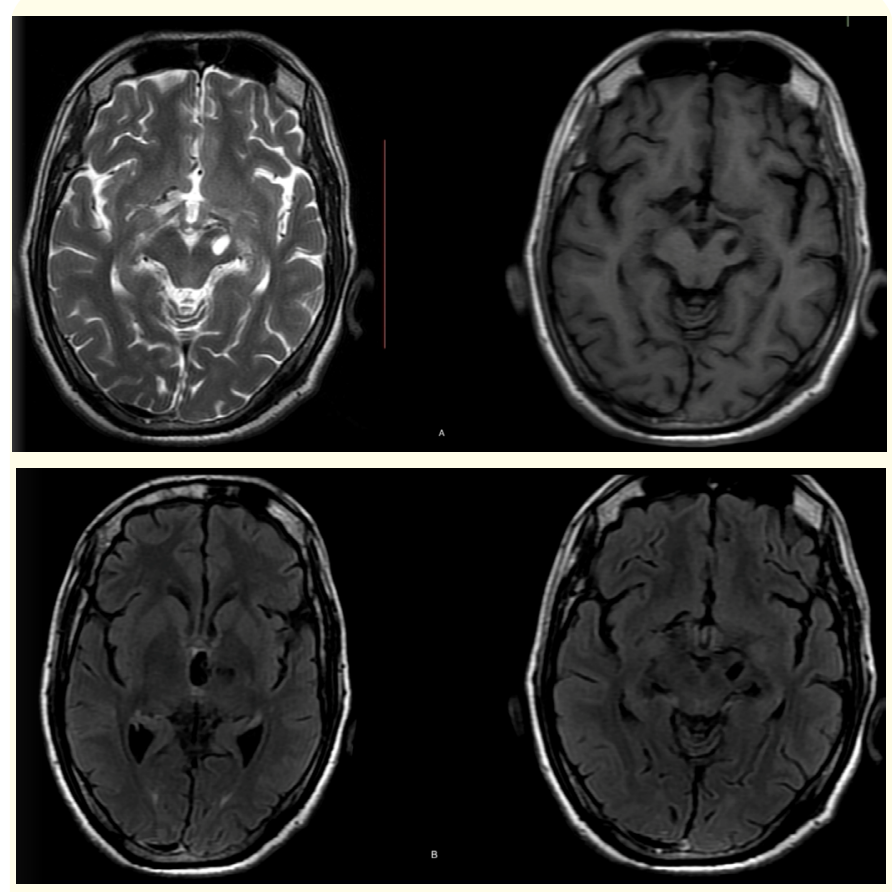

Figure 7a and 7b: Adult male patient presented with seizures. (A) Axial T2W and T1W images (B) Axial FLAIR images show Virchow Robin space in the ponto-mesencephalic junction suggestive of expansion. The VRS appears hyperintense on T2W and hypointense on T1W and FLAIR images. No evidence of FLAIR hyperintense rim. (B) Another enlarged VR space is also noted in the left gangliocapsular region.

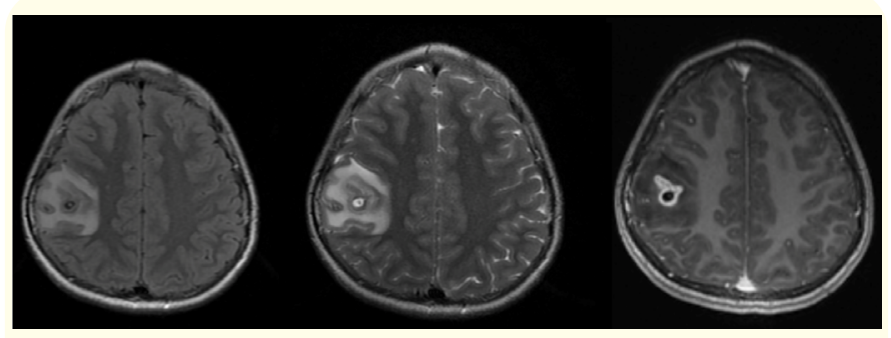

Figure 8: Axial FLAIR, T2W and post contrast T1W images show a well-defined, thick walled, peripherally enhancing lesion with perilesional oedema in the right parietal lobe. These appear hyperintense on T2W and hypointense on FLAIR, with a central T2W hypointense and FLAIR hyperintense dot, suggestive of scolex. On post contrast study, few smaller similar morphology lesions are also noted.
In the granular nodular stage, the walls become thick and show peripheral enhancement on post contrast study. Surrounding parenchymal oedema reduces. During these stages the lesions follow CSF intensity on MR sequences.

In the nodular calcified stage, the lesion calcifies, appearing hypointense on both T1W and T2W sequences. Gradient images are crucial to demonstrate the calcified scolex $[39,40]$.

In comparison to tuberculomas, it is generally smaller in size (less than $10 \mathrm{~mm}$ ), can be solitary, found predominantly in the grey-white matter junction and may involve the ocular and subcutaneous muscles. MR Spectroscopy shows amino acid peaks.

\section{Tuberculoma}

Secondary involvement of the central nervous system is seen frequently. The lesions are usually larger $(>2 \mathrm{~cm})$, with predominance in the posterior fossa. The lesions are associated with meningitis (meningeal enhancement on post contrast study) and hydrocephalus.

Tuberculomas are seen as $\mathrm{T} 2 \mathrm{~W}$ hypointense lesions with ringlike/peripheral enhancement on post contrast images. Lesions are usually multiple (Figure 9). Tuberculomas may resemble neurocysticercosis. However, there is conglomerate enhancement, no scolex is noted, and MR spectroscopy shows lipid peaks.

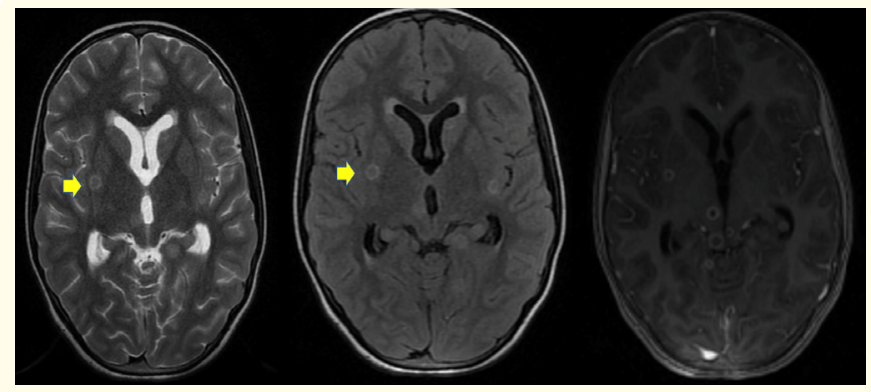

Figure 9: Axial T2W, FLAIR and post contrast T1W image showing multiple round well-defined, peripheral enhancing lesions in bilateral fronto-parietal cortex, and in the posterior fossa. No scolex is noted. The lesions are relatively hypointense, as compared to CSF, on T2W images. 
Cystic neoplasms and metastasis

Giant dilated perivascular spaces can take on bizarre configurations, cause significant mass effect with secondary brain parenchymal loss, and may mimic cystic neoplasms, such as cystic glioblastoma, pilocytic astrocytoma and cystic metastasis (solitary or multiple).

On imaging, most neoplastic etiology lesions are hypointense on T1W and hyperintense on T2W images. However, on FLAIR images, they may not always follow CSF intensity. Most neoplasms have solid components that show significant enhancement on post-contrast images. They also show restriction on diffusion weighted images, a feature not seen with dilated perivascular spaces. However, in case of diagnostic dilemma, it is advisable to carry out follow-up MRI to see the imaging changes.

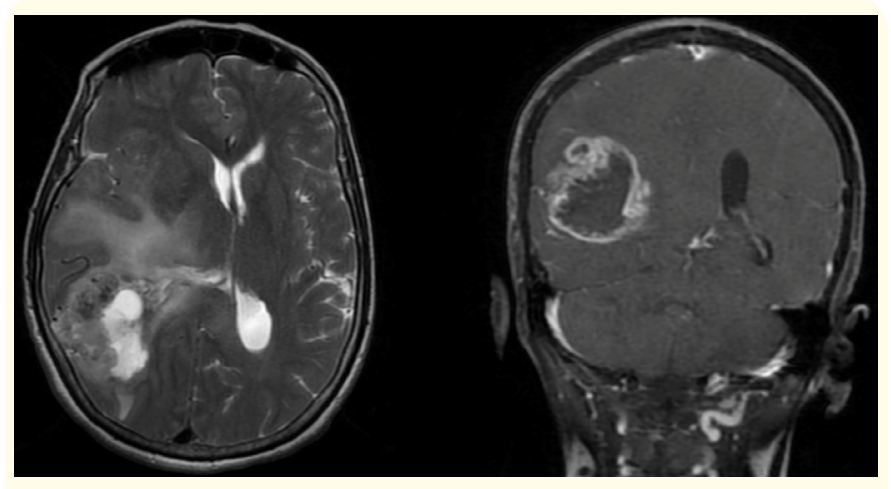

Figure 10: Axial T2W and coronal T1W post-contrast study. A large well-defined, irregularly peripheral enhancing heterogeneous signal intensity lesion with multiple cystic areas and an enhancing soft tissue component within is noted in the right parieto-temporal region. Mass effect in the form of compression and displacement of the adjacent lateral ventricle with midline shift, subfalcine herniation, and perilesional oedema. On diffusion weighted images, the lesion showed restriction, and on gradient images blooming was noted. Presence of enhancing soft tissue component points towards neoplastic aetiology.
Brain cysts

\section{Arachnoid cysts}

These are intra-arachnoid CSF containing cysts that do not have any connection with the ventricular system. Some of these cysts may grow to large sizes and cause mass effect. No post contrast enhancement is noted. The cysts are usually well-defined and follow CSF intensity on all MR sequences. They are differentiated from VR spaces by their typical location: middle cranial fossa, peri-sellar cisterns, and subarachnoid space over convexities [41] (Figure 11).

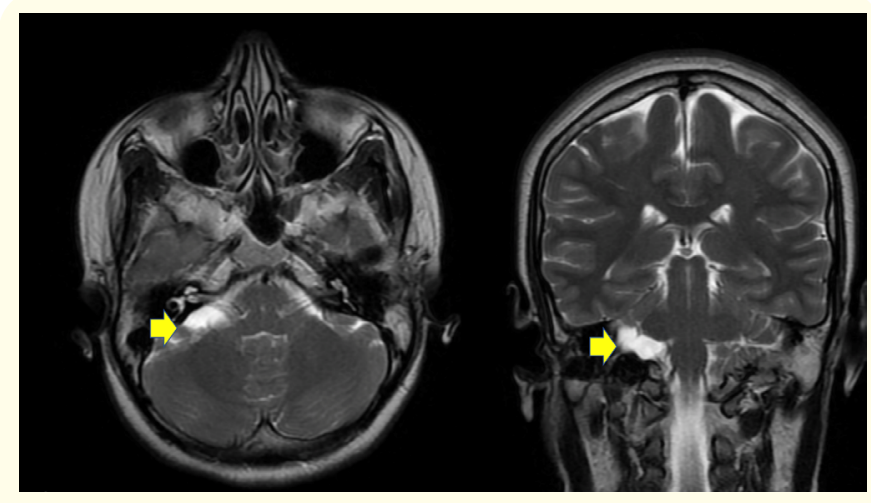

Figure 11: Axial and coronal T2W images shows a CSF intensity $\mathrm{T} 2 \mathrm{~W}$ hyperintense cystic lesion in the right cerebellopontine angle. There was no enhancement on post contrast study. The lesion is seen abutting the right 7th and 8th nerve complexes. No extension into adjacent structures or communication with the ventricular system is noted.

\section{Porencephalic cysts}

These are CSF containing cavities within the brain parenchyma that communicate with the ventricular system directly. It is a relatively rare condition, occurring secondary to vascular occlusion or ischemic insult during foetal development.

These cysts are well-defined and follow CSF intensity on all sequences i.e. hyperintense on T2W and hypointense on T1W and FLAIR images. No post-contrast enhancement is noted. Usually, no mass effect is seen, however, some larger cysts can cause mass effect, symptoms and subsequent adjacent parenchymal atrophy (Figure 12). 


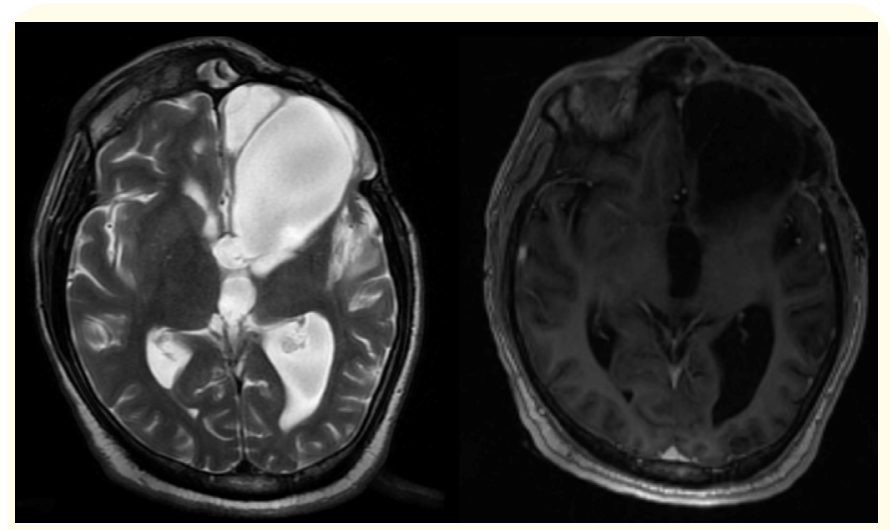

Figure 12: Axial T2W and T1W images showing a CSF intensity cystic lesion involving the grey and white matter of the left frontoparieto-temporal lobes with prominence of adjacent cortical sulci, severe white matter loss and secondary ex-vacuo dilatation of the left lateral ventricle. Communication of the cystic structure with the ventricular system is noted, suggestive of porencephalic cyst.

\section{Isolated sulphite oxidase deficiency (ISOD)}

This is rare inherited autosomal disorder that leads to neurological deterioration and early death, with very few reported cases in literature $[42,43]$. Patients present with seizures and neurological deterioration, usually starting soon after birth.

Neuroimaging findings include atrophy of gyri, cavitation of the centrum semiovale and increased ex-vacuo dilatation of ventricles. In the sulci, marked neuronal loss and gliosis is noted. Loss of white matter myelination, and cavitary changes within white matter is noted $[42,43]$. Features are similar to cystic PVL, and biochemical testing is required to differentiate the two conditions.

\section{Mucopolysaccharidosis (MPS)}

These are a group of inherited disorders with deficiency of enzymes required to break down the mucopolysaccharides (long chain sugar molecules) that are found throughout the body. This subsequently leads to accumulation of intracellular toxic build-up, predominantly glycosaminoglycan (GAG).

The accumulated GAG leads to dilatation of the perivascular spaces and brain atrophy, which results in cribriform appearance of white matter, corpus callosum, basal ganglia on T1W images [21]. On T2W and FLAIR images, the dilated perivascular spaces appear isointense to CSF spaces. Adjacent hyperintensities in the white matter may be noted due to secondary oedema, gliosis or de- or dysmyelination [21] and helps to differentiate from normal VR spaces. Additional findings in the form of breaking of vertebral bodies, widening of the base of skull angle suggestive of platybasia, basilar invagination of cerebellar tonsils, and epiphyseal widening of the long tubular bones of the hand and feet is also noted in MPS IV (Figures 13-15).

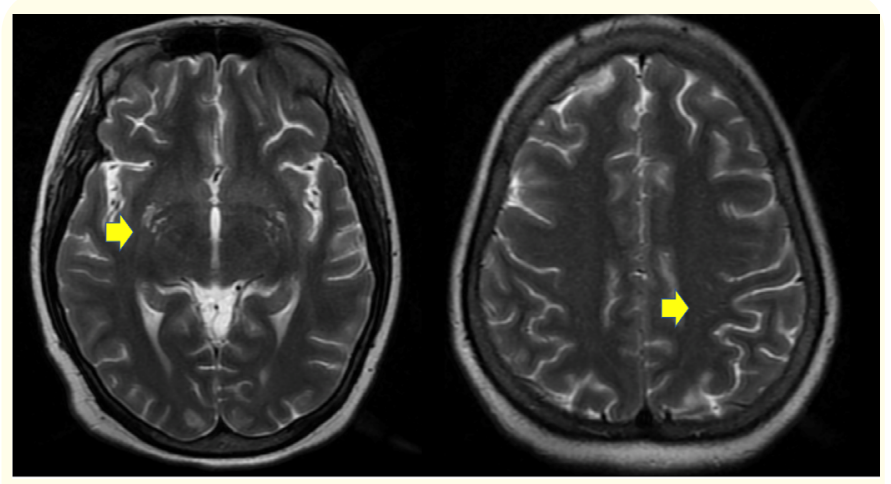

Figure 13: T2W axial images in a patient with Type I MPS (Hurler's syndrome) showing presence of $\mathrm{T} 2 \mathrm{~W}$ hyperintense prominent perivascular spaces along the vascular territories in the subcortical white matter and the ganglio-capsular regions.

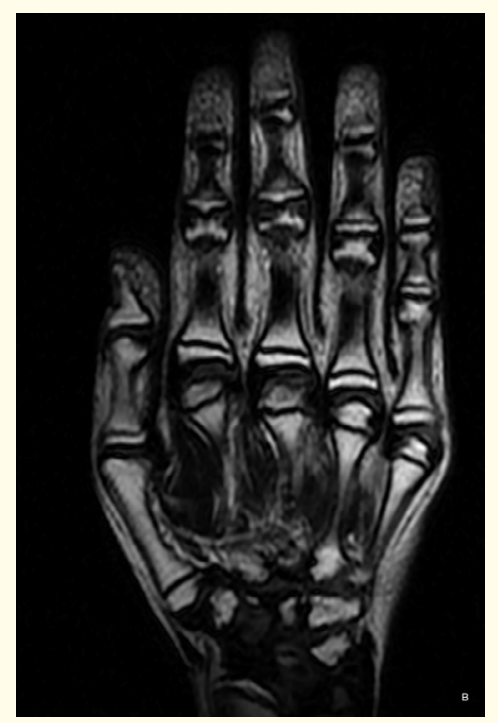

Figure 14: Sagittal T2W image of the spine in a known case of MPS IV (Morquio syndrome) showing breaking of vertebral bodies, involving the dorso-lumbar vertebrae with significant reductio in their height. This is almost pathognomonic from MPS. 


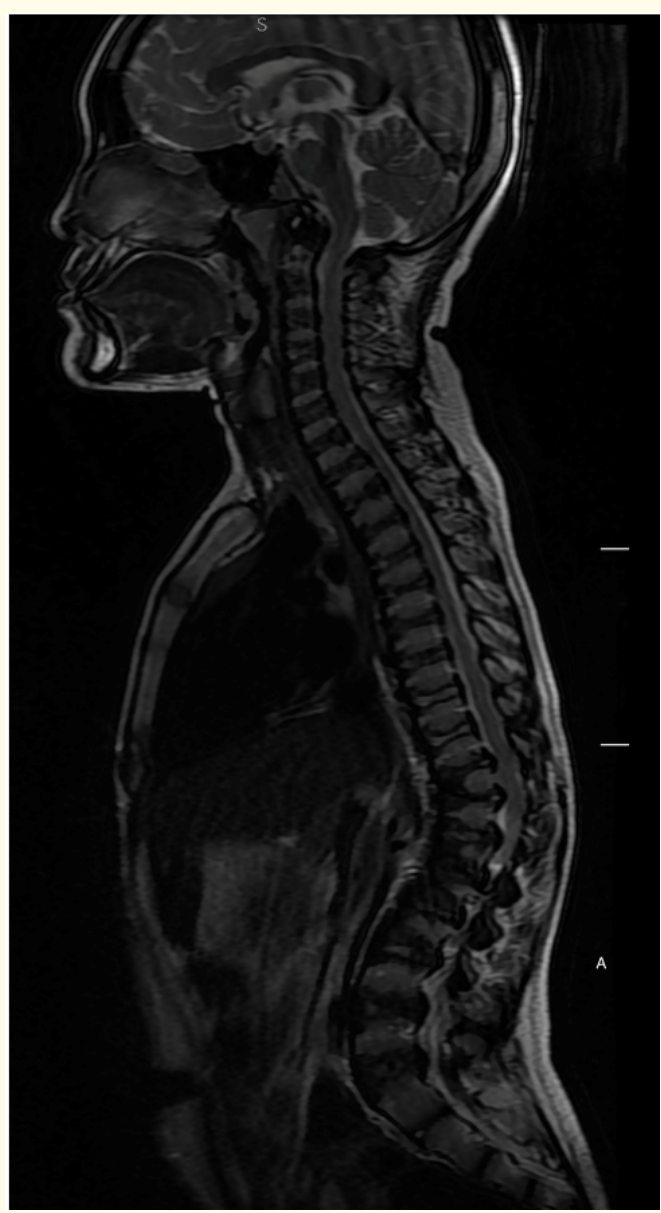

Figure 15: T2W coronal section of right hand showing metaphyseal flaring, wide metacarpals, multiple epiphyseal centres, and wide tubular bones. Proximal metacarpals are pointed.

\section{Conclusion}

Enlarged Virchow-Robin or perivascular spaces are found in the brain parenchyma, distributed along the vascular channels, and can be found at any age. Careful assessment of imaging findings, physical examination and biochemical testing is required to diagnose patients correctly and treat appropriately. The neuroimaging features mentioned above will help to delineate the major mimics of enlarged perivascular spaces.

\section{Acknowledgements}

Dr. Sumit Pruthi: For guiding through the paper outline and authorship

\section{Conflict of Interest}

None.

\section{Bibliography}

1. Ozturk MH and Aydingoz U. "Comparison of MR signal intensities of cerebral perivascular (Virchow-Robin) and subarachnoid spaces". Journal of Computer Assisted Tomography 26 (2002): 902-904.

2. Song CJ., et al. "MR imaging and histologic features of subinsular bright spots on T2-weighted MR images: Virchow-Robin spaces of the extreme capsule and insular cortex". Radiology 214 (2000): 671-677.

3. Pollock H., et al. "Perivascular spaces in the basal ganglia of the human brain: their relationship to lacunes". Journal of Anatomy 191 (1997): 337-346.

4. Heier LA., et al. "Large Virchow-Robin spaces: MR-clinical correlation". American Journal of Neuroradiology 10 (1989): 929936.

5. Karen L Salzman., et al. American Journal of Neuroradiology 26.2 (2005): 298-305.

6. Hutchings M and Weller RO. "Anatomical relationships of the pia mater to cerebral blood vessels in man". Journal of Neurosurgery 65 (1986): 316-325.

7. Zhang ET., et al. "Interrelationship of the pia mater and the perivascular (Virchow-Robin) spaces in the human cerebrum". Journal of Anatomy 170 (1990): 111-123.

8. Schley D., et al. "Mechanisms to explain the reverse perivascular transport of solutes out of the brain". Journal of Theoretical Biology 238 (2006): 962-974.

9. Homeyer P., et al. "A special form of cerebral lacunae: expanding lacunae". Journal of Neurology, Neurosurgery, and Psychiatry 61 (1996): 200-202. 
10. Mascalchi M., et al. "Expanding lacunae causing triventricular hydrocephalus: report of two cases". Journal of Neurosurgery 91 (1999): 669-674.

11. Poirier J., et al. "Thalamic dementia: expansive lacunae of the thalamo-paramedian mesencephalic area-hydrocephalus caused by stenosis of the aqueduct of Sylvius [in French]". Review on Neurology (Paris) 139 (1983): 349-358.

12. Benhaiem-Sigaux N., et al. "Expanding cerebellar lacunes due to dilatation of the perivascular space associated with Binswanger's subcortical arteriosclerotic encephalopathy". Stroke 18 (1987): 1087-1092.

13. Hughes W. “Origin of lacunes”. Lancet 2 (1965): 19-21.

14. Awad IA., et al. "Incidental subcortical lesions identified on magnetic resonance imaging in the elderly". II. Postmortem pathological correlations". Stroke 17 (1986): 1090-1097.

15. Braffman BH., et al. "Brain MR: pathologic correlation with gross and histopathology. 1. Lacunar infarction and VirchowRobin spaces". AJR American Journal of Roentgenology 151 (1988): 551-558.

16. Demaerel P., et al. "Widening of Virchow-Robin spaces". AJNR American Journal of Neuroradiology 17 (1996): 800-801.

17. Ruggieri $\mathrm{M}$ and Pavone L. "Hypomelanosis of Ito: clinical syndrome or just phenotype?" Journal of Child Neurology 15 (2000): 635-644.

18. Ruiz-Maldonado R., et al. "Hypomelanosis of Ito: diagnostic criteria and report of 41 cases". Pediatric Dermatology 9 (1992): 1-10.

19. Groeschel S., et al. "Virchow-Robin spaces on magnetic resonance images: Normative data, their dilatation, and a review of the literature". Neuroradiology 48 (2006): 745-54.

20. Mohan S., et al. "Virchow-Robin spaces in health and disease". Neuroradiology 22 (2009): 518-524.

21. Kwee RM and Kwee TC. "Virchow-Robin spaces at MR imaging”. Radiographics 27 (2007): 1071-1086.
22. Coward RJ., et al. "Kidney disease in hypomelanosis of Ito". Nephrology Dialysis Transplantation 16 (2001): 1267-1269.

23. Adriana S de Almeida., et al. "Hypomelanosis of Ito, neurocutaneous syndromes, incontinentia pigmenti achromians". Journal of Pediatrics (Rio J) 77.1 (2001): 59-62.

24. Phadke RV., et al. Multicystic encephalomalacia secondary to head trauma". Journal of Neurosciences in Rural Practice 8.1 (2017): 158-159.

25. https://www.eurorad.org/case/1095

26. Garten L., et al. "Progressive multicystic encephalopathy: Is there more than hypoxia-ischemia?" Journal of Child Neurology 22 (2007): 645-649.

27. Alam A and Sahu S. "Magnetic Resonance Imaging in Evaluation of Periventricular Leukomalacia”. Medical Journal Armed Forces India 66.4 (2010): 374-380.

28. Schouman-Claeys E., et al. "Periventricular leukomalcia: correlation between MR imaging and autopsy findings during the first two months of life". Radiology 189 (1993): 59-64.

29. Hill A., et al. "Haemorrhagic periventricular leukomalacia: diagnosis by real time ultrasound and correlation with autopsy findings". Paediatrics 69 (1982): 282-284.

30. Siea Lilian T., et al. "Early MR Features of Hypoxic-ischemic brain injury in neonates with periventricular densities on sonograms". American Journal of Nemoradiology 21 (2000): 852861.

31. Prayson RA. "Neuropathology: A Volume in the Series: Foundations in Diagnostic Pathology (Expert Consult - Online and Print)". 2e. Saunders.

32. Elster Ad and Richardson DN. "Focal high signal on MR scans of the midbrain caused by enlarged perivascular spaces: MRpathologic correlation". AJNR American Journal of Neuroradiology 11 (1991): 1119-112.

33. Heier LA., et al. "Large Virchow-Robin spaces: MR-clinical correlation”. AJNR American Journal of Neuroradiology 10 (1998): 929-936. 
34. Jungreis CA., et al. "Normal perivascular spaces mimicking lacunar infarction: MR imaging”. Radiology 169 (1988): 101104.

35. Sasaki M., et al. "Hippocampal sulcus remnant: potential cause of change in signal intensity in the hippocampus". Radiology 188 (1993): 743-746.

36. Duvernoy HM. "Human Brainstem Vessels". Berlin: SpringerVerlag (1978): 16-66.

37. Saeki N., et al. "Microsurgical anatomy of the upper basilar artery and the posterior circle of Willis". Journal of Neurosurgery 46 (1977): 563-578

38. Matlung SE., et al. "Multicystic encephalomalacia as an endstage finding in abusive head trauma". Forensic Science, Medicine, and Pathology 7.4 (2011): 355.

39. do Amaral LL., et al. "Neurocysticercosis: evaluation with advanced magnetic resonance techniques and atypical forms". Topics in Magnetic Resonance Imaging 16 (2005): 127-144.

40. Dumas JL., et al. "Parenchymal neurocysticercosis: follow-up and staging by MRI”. Neuroradiology 39 (1997): 12-18.

41. Van Tassel P and Cure JK. "Nonneoplastic intracranial cysts and cystic lesions". Seminars in Ultrasound, CT and MRI 16 (1995): 186-211.

42. Johnson JL and Duran M. "Molybdenum cofactor deficiency and isolated sulfite oxidase deficiency". In: Scriver CR, Deau$\operatorname{det}$ AL, Sly WS, Valle D, eds. The Metabolic and Molecular Basis of Inherited Disease. $8^{\text {th }}$ edition. New York, NY: McGraw-Hill (1995): 2271-2283.

43. Rupar CA., et al. "Isolated sulfite oxidase deficiency". Neuropediatrics 27 (1996): 299-304.

44. Jeffrey D., et al. "Neuroimaging of Dilated Perivascular Spaces: From Benign and Pathologic Causes to Mimics". Journal of Neuroimaging 28.2 (2018): 139-149.

Volume 4 Issue 6 June 2021

(c) All rights are reserved by Bhavini Gupta., et al. 